\title{
ACÚMULO DE FORRAGEM DURANTE O PERÍODO DE OCUPAÇÃO DOS ANIMAIS EM PASTOS SOB LOTAÇÃO INTERMITENTE
}

\section{FORAGE ACCUMULATION DURING THE STOCKING PERIOD ON PASTURES SUBJECTED TO INTERMITTENT STOCKING}

\author{
Jaciara Diavão ${ }^{1}$ \\ Daniel Schmitt ${ }^{*}$ \\ Cauby Medeiros-Neto ${ }^{1}$ \\ Clovis David Medeiros Martins ${ }^{1}$ \\ André Fischer Sbrissia ${ }^{1}$ \\ 1 Universidade do Estado de Santa Catarina, Lages, SC, Brasil. \\ *Autor para correspondência - daniel.schmitt@veterinario.med.br
}

\section{Resumo}

O objetivo deste trabalho foi avaliar o acúmulo de forragem durante o período de ocupação pelos animais em pastos de capim-quicuiu (Pennisetum clandestinum Hochst. Ex. Chiov.) manejados sob lotação intermitente. Os tratamentos consistiram de quatro proporções de desfolhação (remoção de 40, 50, 60 e 70\% da altura inicial) partindo de uma mesma altura em pré-pastejo $(20 \mathrm{~cm})$. Foram avaliadas: taxas de alongamento foliar e colmos e senescência de folhas, índice de área foliar e taxa de acúmulo de forragem. Adicionalmente, dez perfilhos por faixa foram avaliados para as mesmas variáveis dentro de gaiolas de exclusão. Os efeitos linear, quadrático e cúbico da severidade de desfolhação foram avaliados por contrastes de polinômios ortogonais com 5\% de nível de significância. Houve relação linear e inversa entre a porcentagem do índice de área foliar removido e o alongamento de lâminas foliares e o acúmulo de forragem. As taxas de alongamento de folhas em perfilhos bem como o acúmulo de forragem nas áreas excluídas do pastejo foram maiores em relação aos pastos submetidos a desfolhação. Durante o período de rebaixamento de pastos manejados sob lotação intermitente ocorre acúmulo de forragem e o mesmo é linear e inversamente associado à proporção da área foliar removida.

Palavras-chave: fluxo de tecidos; índice de área foliar; produção de forragem; severidade de desfolhação; taxa de acúmulo.

\begin{abstract}
The aim of this study was to evaluate the forage accumulation during the stocking period on Kikuyu grass pastures (Pennisetum clandestinum Hochst. Ex. Chiov) subjected to intermittent stocking. Treatments consisted of four defoliation levels (removal of 40,50,60, and $70 \%$ relative to the initial height) starting from the same pre-grazing height $(20 \mathrm{~cm})$. The following variables were evaluated: leaf and stem elongation rates (evaluated during four times across the period of occupation), leaf area index, and forage accumulation rates. Additionally, ten tillers/strip were evaluated for the same
\end{abstract}


variables into exclusion cages. The linear, quadratic and cubic effects of severity of defoliation were evaluated by orthogonal polynomials contrasts with $5 \%$ of significance level. There was a linear and inverse relationship between the proportion of removed leaf area index with leaf elongation and herbage accumulation rate. The leaf elongation rate in individual tillers and the forage accumulation rates of tillers on excluded areas from grazing were greater than those under grazing. During the stocking period on pastures submitted to intermittent grazing, forage accumulation was observed and it was linear and negatively correlated with the proportion of leaf area removed.

Keywords: accumulation rate; forage production; leaf area index; severity of defoliation; tissue turnover.

Recebido em: 17 maio de 2016

Aceito em: 16 janeiro de 2017

\section{Introdução}

Um tema relativamente pouco abordado como fim de pesquisa é a determinação do acúmulo de forragem durante o período de ocupação dos animais em pastos manejados sob lotação intermitente. Isso surge da dificuldade para tal avaliação, uma vez que os períodos de ocupação são geralmente curtos, o que restringe o uso de técnicas como gaiolas de exclusão, por exemplo. Outra alternativa seria a determinação do acúmulo por meio da técnica de fluxo de tecidos; no entanto, as avaliações teriam de ser realizadas com alta frequência uma vez que os animais em pastejo podem remover material acumulado antes que o crescimento das folhas possa ser detectado.

Desse modo, pesquisadores têm presumido que o acúmulo de forragem durante o período de ocupação pode ser negligenciado se o período de ocupação for curto $^{(1)}$, ou ainda que as taxas de acúmulo são constantes (comparadas aos períodos de rebrotação) durante o período de ocupação ${ }^{(2)}$. Tais afirmativas podem ser questionáveis, uma vez que a base do manejo em pastos sob lotação contínua, por exemplo, é fundamentada na existência de um acúmulo diário de forragem, ou seja na densidade de lotação necessária para que a pastagem seja mantida em determinada meta (e.g. altura, massa), em que todo o material acumulado ao longo do dia é supostamente consumido pelos animais em pastejo $^{(3)}$.

A produção de forragem é um processo contínuo tanto na de planta (fluxo de tecidos) quanto na população (considerando a densidade populacional de perfilhos na área), contudo, extremamente dependente de fatores limitantes à fotossíntese (e.g. temperatura, luminosidade, qualidade de luz), de modo que em intervalos de tempo (ainda que curtos) deveria existir acúmulo de forragem ${ }^{(4)}$. Plantas forrageiras exibem diferentes potenciais produtivos, sendo muitas vezes determinado pelo tipo de planta (e.g. $\mathrm{C}_{3}$ vs $\mathrm{C}_{4}$, gramínea vs leguminosa), condições edafoclimáticas, manejo empregado (adubações, consórcio) e suas interações. Entretanto, independente da condição, o manejo do pastejo é uma das principais ferramentas para maximizar o potencial produtivo das pastagens ${ }^{(4)}$. Isso ocorre porque a desfolhação (independentemente do método - corte ou pastejo) exerce efeito direto no Índice de Área Foliar (IAF) e indireto na capacidade fotossintética das plantas ${ }^{(3,5,6)}$.

O perfilho, considerado a unidade vegetativa básica, pode representar fielmente o que acontece em um ambiente pastoril ${ }^{(4)}$. Nesse sentido, a taxa de acúmulo calculada via técnica de fluxo de tecidos 
pode ser a ferramenta capaz de detectar as variações (ainda que pequenas) de massa de forragem durante períodos de tempo relativamente curtos. Adicionalmente, variações na estrutura do dossel forrageiro geradas ao longo do período de ocupação dos animais devem gerar variações no acúmulo de forragem. Dentro desse contexto, o objetivo deste trabalho foi avaliar o efeito de diferentes severidades de desfolhação no acúmulo de forragem durante o período de ocupação de pastos de capim-quicuiu (Pennisetum clandestinum Hochst Ex. Chiovi) manejados sob lotação intermitente. As hipóteses testadas foram: i) há acúmulo de forragem durante o período de ocupação de pastos manejados sob lotação intermitente; ii) o acúmulo de forragem durante o período de ocupação de pastos manejados sob lotação intermitente possui correlação negativa com a proporção do IAF removido.

\section{Material e Métodos}

O experimento foi realizado no Centro de Ciências Agroveterinárias (CAV), Universidade do Estado de Santa Catarina (UDESC), Lages/SC, durante os meses de abril e maio de 2014. Segundo a classificação de Köppen, o clima da região é subtropical úmido mesotérmico $(\mathrm{Cfb})$, com temperatura média anual variando entre $17-18{ }^{\circ} \mathrm{C}$ e precipitação média anual de $1500 \mathrm{~mm}$ bem distribuídas ao longo do ano. As condições climáticas médias durante o período experimental estão apresentadas na Figura 1.

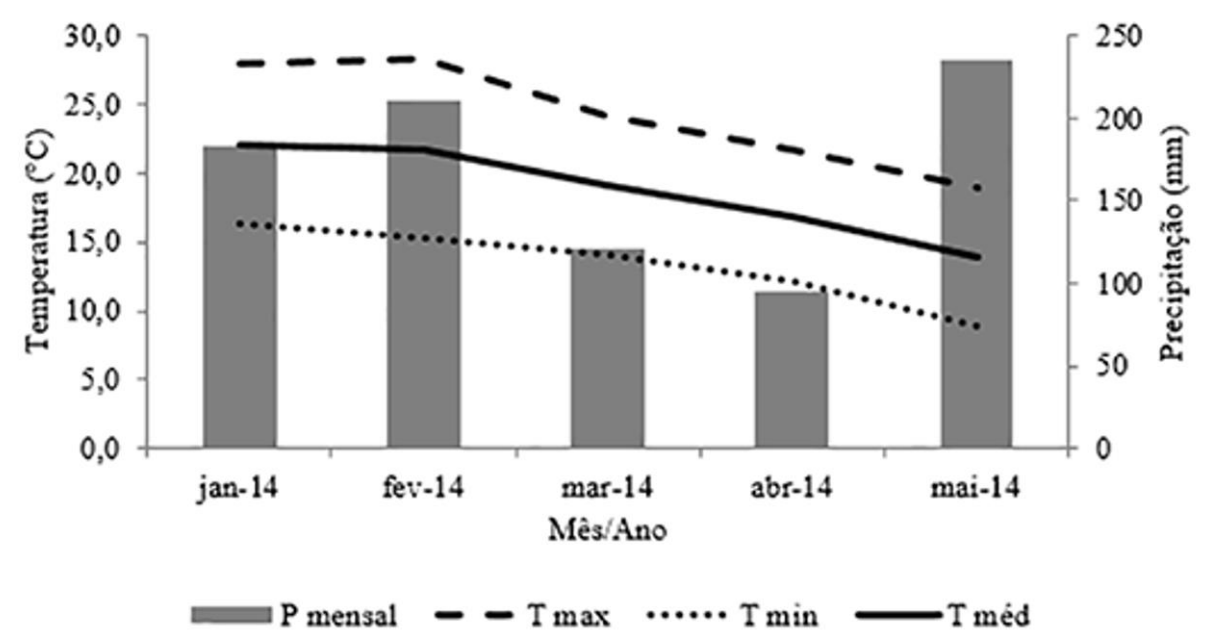

Figura 1. Temperatura máxima média $\left({ }^{\circ} \mathrm{C}\right)$, temperatura minima média $\left({ }^{\circ} \mathrm{C}\right)$, temperatura média $\left({ }^{\circ} \mathrm{C}\right)$, e precipitação mensal $(\mathrm{mm})$ durante o periodo de Janeiro à Junho de $2014 \mathrm{em}$ Lages - SC.

A área experimental era formada de uma pastagem de capim-quicuiu (Pennisetum clandestinum Hochst Ex. Chiovi), que nos últimos anos vinha sendo utilizada em experimentos com lotação intermitente. O solo da área experimental é classificado como Cambissolo Húmico Alumínico Léptico, argiloso, com horizonte A moderado ${ }^{(7)}$. Antes do período experimental, o solo foi amostrado 
na camada de 0-20 cm e sua análise apresentou os seguintes valores: $\mathrm{pH}\left(\mathrm{H}_{2} \mathrm{O}\right) 6,1 ; \mathrm{MO}(\%)$ 2,0; $\mathrm{P}$ (Extrator mehlich, $\left.\mathrm{mg} / \mathrm{dm}^{3}\right)$ 14,7; K $\left(\mathrm{mg} / \mathrm{dm}^{3}\right)$ 0,3; $\mathrm{Ca}\left(\mathrm{mg} / \mathrm{dm}^{3}\right) 7,8 ; \mathrm{Mg}\left(\mathrm{cmol}_{\mathrm{c}} / \mathrm{dm}^{3}\right) 3,5 ; \mathrm{H}+\mathrm{Al}$ $\left(\mathrm{cmol}_{\mathrm{c}} / \mathrm{dm}^{3}\right)$ 3,1; $\mathrm{S}\left(\mathrm{cmol}_{\mathrm{c}} / \mathrm{dm}^{3}\right)$ 11,6; T $\left(\mathrm{cmol}_{\mathrm{c}} / \mathrm{dm}^{3}\right)$ 14,7; V (\%) 79,2. Em abril de 2014 uma roçada a $10 \mathrm{~cm}$ de altura foi realizada seguida de uma adubação nitrogenada com o equivalente a $66 \mathrm{~kg}$ N.ha ${ }^{-1}$ na forma de ureia. Logo em seguida, essa área foi dividida com cerca elétrica em três blocos de acordo com topografia, com quatro unidades experimentais (aproximadamente $1500 \mathrm{~m}^{2} \mathrm{cada}$ ).

Para a remoção de diferentes proporções do IAF em pré-pastejo, optou-se por empregar quatro severidades de desfolhação (remoção de 40, 50, 60 e 70\% da altura em pré-pastejo), partindo de uma mesma altura inicial de $20 \mathrm{~cm}$. A altura do dossel forrageiro foi determinada com a técnica do bastão graduado duas vezes por semana em 50 pontos aleatórios por unidade experimental até atingir a meta pretendida (pré-pastejo) ou até após a ocupação da faixa diária (pós-pastejo - descrita abaixo). A altura pré-pastejo foi baseada em um trabalho prévio conduzido na mesma área experimental ${ }^{(8)}$ que indicou que alturas entre 15-25 não afetam a capacidade produtiva de pastos de capim-quicuiu, desde que combinada com severidades moderadas de desfolhação (não superiores à 50\%).

Todas as unidades experimentais foram divididas em faixas (dois por piquete), sendo cada uma delas pastejada pelo período de um dia. Para tanto, amostras de forragem foram coletadas em cinco pontos representativos da área disponível ao pastejo com o auxílio de armações metálicas de $0,1 \mathrm{~m}^{2}$, coletando-se todo o material presente acima do resíduo pretendido da unidade experimental, ou seja, os cortes de massa foram realizados até as alturas de 12,10,8 e $6 \mathrm{~cm}$ para os tratamentos de 40,50, 60 e $70 \%$ de rebaixamento, respectivamente. Logo após a coleta, essas amostras foram pesadas ainda verdes, misturadas com as demais do respectivo piquete e uma subamostra de $100 \mathrm{~g}$ coletada para a determinação do teor de matéria seca (\%MS) em forno de microondas. Finalmente, pelo produto entre $\% \mathrm{MS}$ e a disponibilidade do material verde no estrato pastejável, foi determinada a massa de forragem em pré-pastejo (em kg MS/ha). A densidade de lotação a ser ocupada em cada faixa foi calculada utilizando-se uma oferta de $3 \mathrm{~kg}$ de matéria seca/100 kg de peso vivo animal. Para tanto, foram utilizadas novilhas e vacas secas Holandês X Jersey (Tabela 1).

Tabela 1. Valores médios de densidade de lotação ( $\mathrm{kg}$ PV/faixa), área das faixas $\left(\mathrm{m}^{2}\right)$ e massa de forragem acima do resíduo pretendido ( $\mathrm{kg} \mathrm{MS/ha)} \mathrm{de} \mathrm{pastos} \mathrm{de} \mathrm{capim-quicuiu} \mathrm{submetidos}$ a diferentes proporçôes de rebaixamento

\begin{tabular}{cccc}
\hline Tratamento & Densidade de Lotação & Área da faixa & Massa de Forragem \\
\hline 40 & $1583\left( \pm 194^{*}\right)$ & $236( \pm 56)$ & $2054( \pm 363)$ \\
50 & $1838( \pm 435)$ & $285( \pm 20)$ & $1945( \pm 551)$ \\
60 & $2438( \pm 712)$ & $339( \pm 53)$ & $2147( \pm 528)$ \\
70 & $3080( \pm 753)$ & $321( \pm 13)$ & $2969( \pm 489)$ \\
\hline
\end{tabular}

*Valores entre parênteses correspondem ao desvio padrão.

O acúmulo de forragem durante o período de ocupação ( $\mathrm{kg}$ de $\mathrm{MS} / \mathrm{ha} / \mathrm{dia}$ ) foi determinado por meio do produto entre o crescimento médio por perfilho, a densidade populacional de perfilhos e um fator de conversão ( $\mathrm{cm}$ de folha/g de MS). A metodologia para determinação dessas três variáveis foram as seguintes:

i) $\mathrm{O}$ crescimento médio por perfilho foi determinado por meio da técnica de perfilhos $\operatorname{marcados}^{(5)}$. 
Para tanto, 20 perfilhos representativos da condição média (avaliação visual da altura e massa de forragem) da faixa avaliada foram identificados com fita adesiva em quatro réguas transectas (cinco perfilhos por transecta) distribuídos ao longo da faixa. Cada régua foi alocada transversalmente ao comprimento da faixa e os cinco perfilhos uniformemente distribuídos. Adicionalmente, gaiolas de exclusão foram utilizadas em todas as faixas, onde, em seu interior, dez perfilhos foram marcados para fins de determinação do efeito da ausência do pastejo, durante o mesmo período de tempo, no crescimento dos pastos. As medições tomadas nessa avaliação foram: altura do perfilho estendido (distância entre o solo e o ápice da folha mais alta quando alinhadas verticalmente), comprimento de colmo + pseudocolmo (distância entre o solo e a lígula da última folha completamente expandida) e o comprimento e largura (tomada a partir da porção mediana) de todas as lâminas foliares. Adicionalmente, todas as folhas presentes no perfilho foram classificadas como intacta (sem sinais de corte ou pisoteio) ou desfolhada, completamente expandida (presença da lígula) ou em expansão (lígula não visível) e folhas vivas ou senescentes (ausência/presença de áreas amareladas). Para folhas completamente expandidas o comprimento era tomado a partir da base (lígula) até o ápice da folha; já as em expansão eram medidas a partir da base da última folha com lígula visível. Folhas senescentes eram medidas a partir de sua origem até o início da área em senescência. Perfilhos eventualmente arrancados eram contabilizados e desconsiderados das avaliações subsequentes. Todas as avaliações iniciavam antes da entrada dos animais na faixa (7:00 - 8:00) com a identificação dos perfilhos e classificação das folhas e leitura das alturas/comprimentos. As avaliações subsequentes eram realizadas após a atividade de pastejo da manhã (entre às 11:00 e 12:00), da tarde (após às 18:00 horas) e logo após a retirada dos animais (9:00 horas do dia seguinte). Sendo assim, quatro leituras foram realizadas ao longo de 24 horas de ocupação das faixas.

ii) A densidade populacional de perfilhos (DPP) foi determinada pela contagem dos perfilhos contidos em armações metálicas com $0,0625 \mathrm{~m}^{2}$ de área e dispostos em três pontos representativos da condição média da faixa no momento da coleta (visualmente determinada). Correções na DPP foram realizadas para determinação do número de perfilhos removidos pela ação do pastejo. Para tanto, considerou-se que cada perfilho arrancado representava $5 \%$ da população total (uma vez que haviam 20 perfilhos marcados por unidade experimental) e que, na média, o arranquio ocorreria como um evento aleatório e normalmente distribuído ao longo do tempo.

iii) $\mathrm{O}$ fator de conversão foi gerado por meio da relação entre o tamanho e o peso do componente

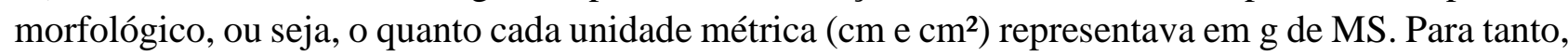
uma amostra de perfilhos que representava a condição média em pré-pastejo da faixa foi coletada. $\mathrm{O}$ corte foi efetuado ao nível do solo e foram medidos individualmente o comprimento e a largura das lâminas foliares (de diferentes categorias) e apenas o comprimento de colmo + pseudocolmo. Após medidos, todos os componentes foram acondicionados em sacos de papel devidamente identificados e levados à estufa com circulação forçada de ar a $65^{\circ} \mathrm{C}$ por 72 horas para determinação de seus respectivos pesos secos. Finalmente, o quociente entre peso e o tamanho do componente morfológico gerou o fator de conversão (g de MS/cm e/ou g de MS/cm²).

iv) A taxa de acúmulo de forragem durante o período de ocupação foi quantificada por meio do produto entre o crescimento médio de lâminas foliares por perfilho, a densidade populacional de perfilhos e o fator de conversão $(1 \mathrm{~cm}$ de folha $=0,001279 \mathrm{~g}$ de $\mathrm{MS}$ ) e convertido para $\mathrm{kg}$ de $\mathrm{MS} / \mathrm{ha} / \mathrm{dia}$.

O índice de aérea foliar em pré e pós-pastejo foi determinado de maneira indireta em três etapas:

i) Amostras de forragem (10/faixa) foram coletadas em pré e pós-pastejo com o auxílio de armações metálica de $0,1 \mathrm{~m}^{2}$ e corte sendo efetuado rente ao solo. Todo o conteúdo morfológico foi separado 
(lâminas foliares, colmo + pseudocolmo, material morto e plantas invasoras) e as folhas contidas nessa amostra foram pesadas e acondicionadas em estufa de circulação forçada de ar a $65^{\circ} \mathrm{C}$ por 72 horas.

ii) 660 lâminas foliares verdes oriundas de 100 perfilhos tiveram sua porção senescente destacada (quando presente) e a área foliar determinada através da relação entre o comprimento e largura medida com régua graduada em milímetros $\left(\mathrm{AF}_{\mathrm{C}}\right)$ e, posteriormente, medidas em integrador de área foliar (modelo LICOR 3000). Esse último método foi considerado como o a área foliar "real" ( $\left.\mathrm{AF}_{\mathrm{R}}\right)$.

iii) Com base nos valores de massa de lâminas foliares $\left(\mathrm{kg} \mathrm{MS} / \mathrm{m}^{2}\right)$ e o fator de conversão descrito acima $\left(0,00298 \mathrm{~g}=1 \mathrm{~cm}^{2}\right.$ de folha), foi possível calcular a $\mathrm{AF}_{\mathrm{C}}$, que pode ser convertido para $\mathrm{AF}_{\mathrm{R}}$ (Figura 2) e posteriormente ao IAF (a partir da multiplicação pela DPP).

Os dados foram arranjados como média das unidades experimentais (a partir das duas réplicas) e submetidos à análise de variância através do procedimento GLM (General Linear Models) do pacote estatístico SAS (Statistical Analysis System), versão 9.0 para Windows (SAS, 1999). Os efeitos linear, quadrático e cúbico da severidade de desfolhação foram testados por meio de contrastes de polinômios ortogonais, sendo os coeficientes gerados pelo procedimento IML (Interactive Matrix Language) do pacote estatístico SAS. Esse procedimento foi adotado uma vez que os tratamentos efetivamente alcançados não foram equidistantes entre si. Quando conveniente, relações funcionais (modelos lineares) foram geradas com o intuito de se facilitar a visualização dos dados obtidos. $\mathrm{O}$ nível de significância adotado para todo o experimento foi de $5 \%(\mathrm{P}<0,05)$.

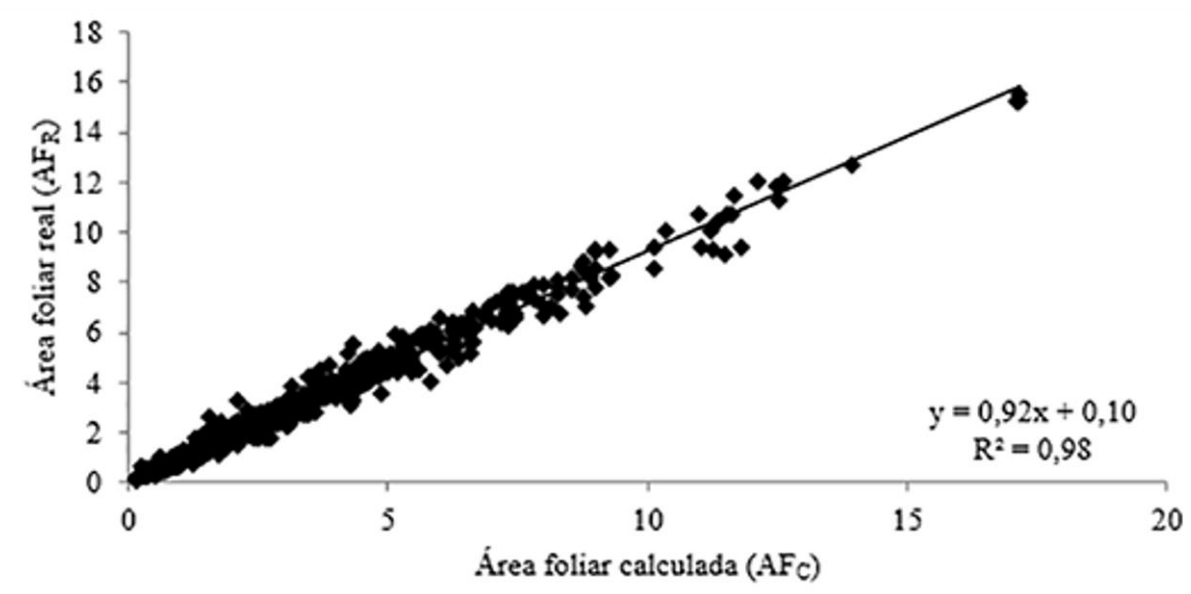

Figura 2. Relação entre área foliar calculada por meio da multiplicação entre comprimento e largura e a área foliar real (obtida por meio de integrador de área foliar LI-3100C) em pastos de capim-quicuiu manejados sob lotação intermitente.

\section{Resultado e Discussão}

As metas de severidades pré-estabelecidas não foram efetivamente alcançadas. Entretanto, houve uma redução linear $(\mathrm{P}<0,05)$ da altura em pós-pastejo, de modo que os dados da Tabela 2 foram analisados em função da severidade efetivamente alcançada nos tratamentos pré-estabelecidos. A dificuldade em se alcançar as metas de alturas pós-pastejo esteve associada à crescente presença de 
colmos ao longo do processo de rebaixamento da pastagem ${ }^{(9)}$, estrutura que impõe dificuldades ao processo de forrageamento pelos animais ${ }^{(10-14)}$ e que podem ter sido inadvertidamente tocados durante a leitura das alturas, gerando valores subestimados da profundidade de pastejo. Adicionalmente, um fator interessante que pode ter contribuído para esse problema foi o comportamento dos animais, que pastejaram principalmente áreas próximas as cercas divisórias, deixando áreas rejeitadas (por fezes, urina e pisoteio) na área central das faixas.

Como esperado, as variáveis referentes à estrutura em pré-pastejo (massa de forragem, DPP e IAF) não foram diferentes entre os tratamentos ( $\mathrm{P}>0,05$ - Tabela 2) uma vez que todas as unidades experimentais partiram de uma mesma altura inicial $(20 \mathrm{~cm})$. Entretanto, os valores de massa de forragem, DPP e IAF em pós-pastejo (Tabela 2) não apresentaram diferenças significativas ( $\mathrm{P}>0,05)$, ainda que as alturas em pós-pastejo tenham reduzido em função dos tratamentos. Segundo Hodgson ${ }^{(3)}$, a altura do dossel forrageiro possui forte relação com características do dossel forrageiro como massa de forragem, relação lâmina:colmo, IAF e IL, sendo um critério confiável na criação e manutenção de estruturas de dosséis forrageiros. Nesse sentido, a provável razão para a ausência de diferenças para massa de forragem em pós-pastejo se deve à natureza variável desse tipo de dado, o que gera grandes desvios e dificuldades em análises estatísticas convencionais. Ademais, isso explicaria a ausência de diferenças significativas dos valores de IAF em pós-pastejo, uma vez que estes foram estimados a partir dos valores de massa de forragem. Por fim, a ausência de variação na DPP era uma reposta esperada pois o período de ocupação das faixas foi extremamente curto (1 dia) para que novos perfilhos fossem produzidos ou senescidos.

Tabela 2. Altura do dossel $(\mathrm{cm})$, massa de forragem $(\mathrm{kg}$ de $\mathrm{MS} / \mathrm{ha})$, densidade populacional de perfilhos (DPP-perfilhos $/ \mathrm{m}^{2}$ ) e indice de área foliar (IAF) em pré e pós-pastejo em pastos de capim-quicuiu submetidos a diferentes proporções de rebaixamento.

\begin{tabular}{|c|c|c|c|c|c|c|c|c|}
\hline \multirow{2}{*}{ Variável } & \multicolumn{4}{|c|}{ Proporção do rebaixamento } & \multirow{2}{*}{$\mathbf{E P M}^{*}$} & \multicolumn{3}{|c|}{ Efeito\# } \\
\hline & $40 \%$ & $46 \%$ & $48 \%$ & $54 \%$ & & LIN & QUA & CUB \\
\hline \multicolumn{9}{|c|}{ Pré-pastejo } \\
\hline Altura & 19,7 & 20,1 & 19,6 & 19,3 & 0,9 & NS** & NS & NS \\
\hline Massa & 7435 & 5865 & 6319 & 6378 & 416 & NS & NS & NS \\
\hline DPp & 5077 & 5664 & 5680 & 4795 & 464 & NS & NS & NS \\
\hline IAF & 6,1 & 5,0 & 5,3 & 5,8 & 0,4 & NS & NS & NS \\
\hline \multicolumn{9}{|c|}{ Pós-pastejo } \\
\hline Altura & 11,7 & 10,7 & 10,4 & 9 & 0,4 & 0,002 & NS & NS \\
\hline Massa & 5283 & 4086 & 3988 & 4112 & 514 & NS & NS & NS \\
\hline DPp & 4284 & 5111 & 4740 & 3654 & 453 & NS & NS & NS \\
\hline IAF & 1,8 & 1,6 & 1,3 & 1,2 & 0,4 & NS & NS & NS \\
\hline
\end{tabular}

*Erro padrão da média; **N.S.: Não significativo (P>0,05); ; Efeito: Linear (LIN), Quadrático (QUA) e Cúbico (CUB). 
Houve redução na taxa de alongamento de lâminas foliares expressa tanto em valores absolutos quanto como uma proporção do alongamento observado dentro das gaiolas de exclusão com a porcentagem do IAF removido (Figura 3). Padrão de resposta semelhante já havia sido descrito por Mazzanti et al ${ }^{(16)}$, que encontraram reduções na taxa de alongamento foliar de perfilhos desfolhados em relação à não desfolhados em pastos de Festuca arundinacea Schreb. Isso ocorre porque, durante o período de ocupação, grande parte do material de alto potencial fotossintético (folhas em expansão e recém-maduras) é gradativamente colhido sendo o material remanescente composto majoritariamente de folhas velhas/senescentes e colmos, com baixa capacidade fotossintética e pouco adaptadas às condições de alta luminosidade ${ }^{(6,15)}$. Além disso, a desfolhação pode reduzir o alongamento de folhas pelo dano causado em parte dos meristemas ${ }^{(17)}$. Nesse sentido, a magnitude e as taxas de remoção dessas estruturas são determinadas pelos critérios de manejo (severidade de desfolhação e densidade de lotação ${ }^{(15)}$ ). De modo contrário, perfilhos não submetidos ao pastejo possuem meristemas normalmente intactos e aparato fotossintético composto por folhas jovens que assimilam maior quantidade de luz e maximizam a fotossíntese ${ }^{(6)}$. Sendo assim, as taxas de crescimento de folhas são menores que aquelas observadas em perfilhos que não foram pastejados, embora sejam existentes e significativas.

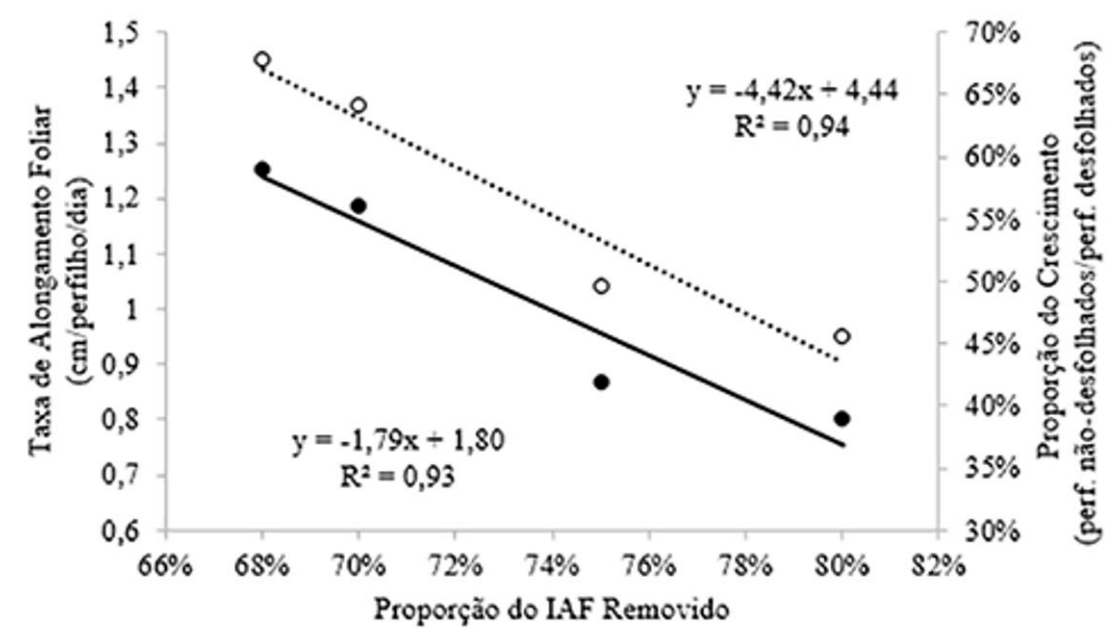

Figura 3. Relação entre o alongamento de lâminas foliares (linha sólida) de perfilhos submetidos ao pastejo e a proporção do alongamento foliar (perfilhos pastejados/perfilhos excluidos do pastejo, avaliados dentro de gaiolas de exclusão) - linha pontilhada) e a porcentagem do indice de área foliar removido (\%) durante a ocupação de pastos de capimquicuiu manejados sob lotação intermitente a partir de uma mesma altura de entrada e diferentes proporções de rebaixamento.

Ao longo do período de avaliação (24 horas), não foram detectadas variações significativas nas taxas de alongamento de colmos ou senescência. Segundo Padilha ${ }^{(8)}$, pastos de capim-quicuiu manejados sob lotação intermitente atingem o IAF crítico em alturas pré-pastejo próximas a $25 \mathrm{~cm}$, condição essa que controla as taxas de alongamento de colmos e senescência e otimiza a taxa de alongamento foliar $^{(4)}$. Adicionalmente, variáveis ligadas à lâmina foliar (e.g. taxa de alongamento e comprimento) são as mais importantes na determinação do acúmulo de forragem de pastos em estádio vegetativo ${ }^{(18)}$.

Os dados absolutos de acúmulo de forragem e o acúmulo expresso como uma proporção do material 
acumulado dentro das gaiolas de exclusão são apresentados na Figura 4. Houve redução linear no acúmulo de forragem em relação à proporção do IAF removido. Interessante observar que mesmo para uma remoção de $80 \%$ do IAF, pastos de capim-quicuiu acumularam durante as 24 horas cerca de $50 \mathrm{~kg}$ de $\mathrm{MS} / \mathrm{ha} /$ dia, o que representou cerca de $40 \%$ do acúmulo observado dentro das gaiolas de exclusão. Em uma das poucas referências sobre o assunto, Dobos et al ${ }^{(2)}$ citou valores médios de acúmulo para capim-quicuiu durante o período de ocupação de aproximadamente $60 \mathrm{~kg} \mathrm{MS} / \mathrm{ha} / \mathrm{dia}$, valores próximos aos observados no presente experimento.

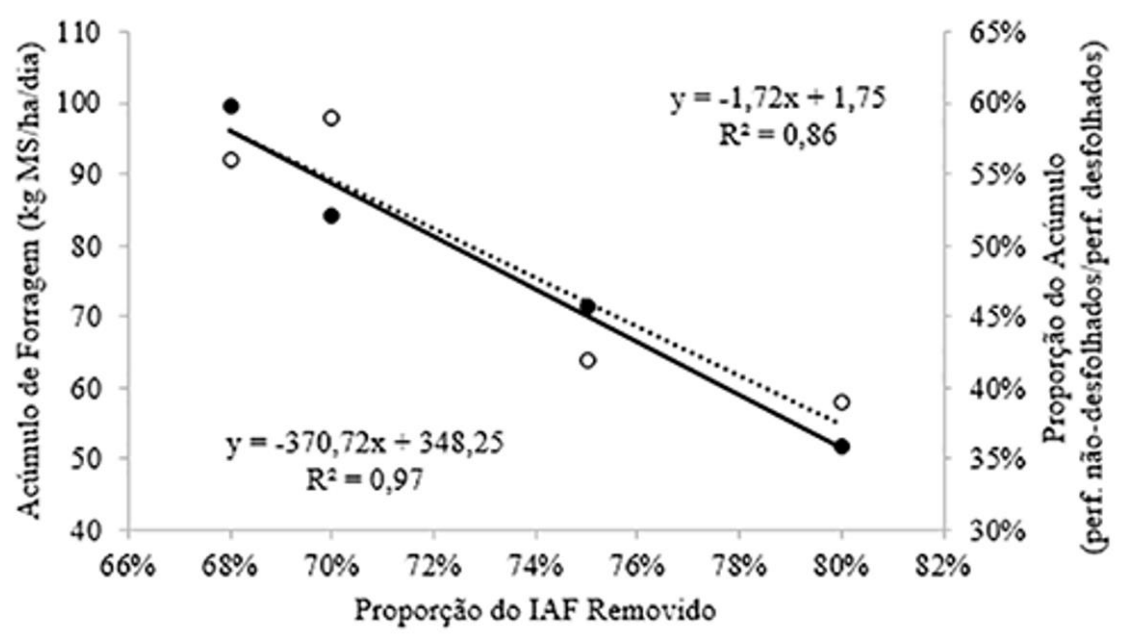

Figura 4. Relação entre o acúmulo de forragem (linha sólida) de perfilhos submetidos ao pastejo e a proporção do acúmulo de forragem (perfilhos pastejados/perfilhos excluídos do pastejo, avaliados dentro das gaiolas de exclusão - linha pontilhada) e a porcentagem do indice de área foliar removido (\%) durante a ocupação de pastos de capim-quicuiu manejados sob lotação intermitente a partir de uma mesma altura de entrada e diferentes proporções de rebaixamento.

Em um primeiro momento, a Figura 4 parece contradizer Parsons et al $^{(6)}$, que afirmaram que desfolhações lenientes não devem ser interpretadas como sinônimos de altas taxas de crescimento, uma vez que seriam acompanhadas de altas taxas de senescência. Entretanto, dois pontos devem ser levados em consideração ao se comparar os experimentos: i) como comentado pelo próprio autor, os seus dados devem ser visualizados como uma resposta após uma desfolhação instantânea, o que não ocorreu no caso do presente experimento, uma vez que o processo de rebaixamento ocorreu de forma gradual ao longo de 24 horas; ii) nos dados de acúmulo do presente experimento foram desconsideradas as taxas de senescência, uma vez que não foi possível detectá-la por meio da técnica empregada. De qualquer forma, ambos os trabalhos convergem para a ideia de que aumentos no IAF removido causam atrasos em se atingir a altas taxas de crescimento.

Uma implicação importante dos dados do presente experimento é que as taxas de acúmulo durante o período de ocupação dos animais em pastos sob lotação intermitente não podem ser negligenciadas uma vez que, mesmo em condições extremas, quando todas as folhas de um perfilho foram removidas, ainda assim ocorreu alongamento foliar. Nesse sentido, ajustes em taxa de lotação devem levar em consideração esse acúmulo e podem assumir uma grande importância no dimensionamento de ocupação das áreas, uma vez que quanto maior o período de ocupação mais significativo seriam os ajustes necessários para que uma determinada meta de pós-pastejo fosse alcançada (e.g. altura, massa, 
IAF). Entretanto, cabe ressaltar que essa condição passaria a ser dependente da área destinada ao pastejo (tamanho de piquete e/ou faixas), uma vez que a produção de forragem em pequenas áreas poderia não permitir, por exemplo, a adição de uma unidade animal extra. Por exemplo, assumindo um acúmulo de forragem de $50 \mathrm{~kg}$ de $\mathrm{MS} / \mathrm{ha} /$ dia durante o período de ocupação dos animais (o menor valor observado no presente experimento), isso significa que em um piquete/faixa de $500 \mathrm{~m}^{2}$ a produção de forragem seria de $2,5 \mathrm{~kg}$ de MS/dia, o que, em tese, não permitiria a alocação de outro animal à área (exceção de pequenos herbívoros). Por outro lado, se o tamanho do piquete for, por exemplo, de 2 ha, o material acumulado seria de $100 \mathrm{~kg} \mathrm{MS} /$ dia, o que permitiria incrementos em até $5000 \mathrm{~kg}$ de PV na área, considerando um consumo de 2\% (expresso em MS) do peso vivo, durante um dado período de ocupação.

\section{Conclusão}

Ocorre acúmulo de forragem durante o período de rebaixamento em pastos manejados sob lotação intermitente e o mesmo é inversamente associado à proporção da área foliar removida.

\section{Referências}

1. Lukuyu M, Paull DR, Johns WH, Niemeyer D, Mcleod J, McCorkell B, Savage D, Purvis IW, Greenwood PL. Precision of estimating individual feed intake of grazing animals offered low, declining pasture availability. Anim Prod Sci. 2014; 54 (12): 2105-2011. http://www.publish.csiro.au/paper/AN14531.htm

2. Dobos RC, Fulkerson WJ, Sinclair K, Hinch GN. Grazing behaviour and pattern of intake of dairy cows grazing kikuyu (Pennisetum clandestinum) grass pastures in relation to sward height and length of grazing session. Anim Prod Sci 2009; 49 (7): 574-585. http://www.publish.csiro.au/paper/EA08212.htm

3. Hodgson J. Grazing management - Science into practice. 1 ed. Essex: Longman Scientific and Technical; 1990. 203 p.

4. Da Silva SC, Sbrissia AF, Pereira LET. Ecophysiology of $\mathrm{C}_{4}$ forage grasses - Understanding plant growth for optimising their use and management. Agriculture. 2015; 5(3): 598 - 625. http://www.mdpi.com/2077$\underline{0472 / 5 / 3 / 598}$

5. Bircham JS, Hodgson J. The influence of sward condition on rates of herbage growth and senescence in mixed swards under continuous stocking management. Grass Forage Sci. 1983; 38 (4): 323 - 331. http://onlinelibrary.wiley.com/doi/10.1111/j.1365-2494.1983.tb01656.x/abstract

6. Parsons AJ, Johnson IR, Harvey A. Use of a model to optimize the interaction between frequency and severity of intermittent defoliation and to provide a fundamental comparison of the continuous and intermittent defoliation of grass. Grass Forage Sci. 1988; $43 \quad$ (1): $49 \quad$ - 59. http://onlinelibrary.wiley.com/doi/10.1111/j.1365-2494.1988.tb02140.x/abstract

7. EMBRAPA - Centro Nacional de Pesquisa de Solos. Sistema brasileiro de classificação de solos. 2 ed. Rio de Janeiro: Embrapa-SPI; 2006. 306 p.

8.Padilha, DA. Acúmulo de forragem e composição química em pastos de capim-quicuiu submetidos a estratégias de lotação intermitente. 2013. 62 p. Dissertação (Mestrado em Ciência Animal) - Centro de Ciências Agroveterinárias, Universidade do Estado de Santa Catarina, Lages. 2013. Disponível em: http://www.cav.udesc.br/arquivos/id_submenu/756/dissertacao_deisy_andrade_padilha1.pdf 
9. Zanini GD, Santos GT, Schmitt D, Padilha DA, Sbrissia AF. Distribuição de colmo na estrutura vertical de pastos de capim Aruana e azevém anual submetidos a pastejo intermitente por ovinos. Ciência Rural. 2012; 42 (5): 882 - 887. http://www.scielo.br/scielo.php?script=sci_arttext\&pid=S0103-84782012000500020

10. Benvenutti MA, Gordon IJ, Poppi DP. The effect of the density and physical properties of grass stems on the foraging behaviour and instantaneous intake rate by cattle grazing an artificial reproductive tropical sward. Grass Forage Sci. 2006; 61 (3): 272 - 281. http://onlinelibrary.wiley.com/doi/10.1111/j.13652494.2006.00531.x/abstract;jsessionid=F722A81A52D31A8B0C2556565DAD122A.d01t01

11. Benvenutti MA, Gordon IJ, Poppi DP. The effects of stem density of tropical swards and age of grazing cattle on their foraging behaviour. Grass Forage Sci. 2008; 63 (1): 1 - 8 . http://onlinelibrary.wiley.com/doi/10.1111/j.1365-2494.2007.00609.x/abstract

12. Prache S, Delagarde R. The influence of vegetation characteristics on foraging strategy and ingestive behaviour. In: Lemaire G, Hodgson J, Chabbi A. Grassland Productivity and Ecosystem Services. 1 ed. Cambridge:CABI; 2011. p. 1585-601.

13. Fonseca L, Mezzalira JC, Bremm C, Filho RSA., Gonda HL, Carvalho PCF. Management targets for maximising the short-term herbage intake rate of cattle grazing in Sorghum bicolor. Livest Sci; 2012; 145 (13): 205 - 211. http://www.sciencedirect.com/science/article/pii/S1871141312000595

14. Mezzalira JC, De Faccio Carvalho PC, Fonseca L, Bremm C, Cangiano C, Gonda HL, Laca EA. Behavioural mechanisms of intake rate by heifers grazing swards of contrasting structures. Appl Anim Behav Sci; 2014; 153 (April): 1 - 9. http://www.appliedanimalbehaviour.com/article/S0168-1591(13)003213/abstract

15. Lemaire G, Silva SC, Agnusdei M, Wade M, Hodgson J. Interactions between leaf lifespan and defoliation frequency in temperate and tropical pastures: a review. Grass Forage Sci. 2009; (4): 341 - 353. http://onlinelibrary.wiley.com/doi/10.1111/j.1365-2494.2009.00707.x/abstract

16. Mazzanti A, Lemaire G, Gastal F. Effects of nitrogen fertilization upon herbage production of tall fescue swards continuously grazed by sheep. 1. Herbage growth dynamics. Grass Forage Sci. 1994; 49(2): 111-120. http://onlinelibrary.wiley.com/doi/10.1111/j.1365-2494.1994.tb01983.x/abstract

17. Gastal F, Lemaire G. Defoliation, shoot plasticity, sward structure and herbage utilization in pasture: review of the underlying ecophysiological processes. Agriculture. 2015; 5(4): 1146 - 1471. http://www.mdpi.com/2077-0472/5/4/1146

18. Barre P, Turner L, Escobar-Gutiérrez A. Leaf length variation in perennial forage grasses. Agriculture. 2015; 5(3): 682-696. http://www.mdpi.com/2077-0472/5/3/682 